\title{
Intervenções de enfermagem nas reações adversas em pacientes oncológicos em uso de imunoterapia: Uma revisão de escopo
}

\author{
Nursing interventions in adverse reactions in cancer patients using immunotherapy: A scoping \\ review
}

Intervenciones de enfermería en reacciones adversas en pacientes oncológicos mediante inmunoterapia: Revisión del alcance

\section{Resumo}

Este estudo tem como objetivo mapear as evidências científicas sobre intervenções de enfermagem relacionadas ao manejo de reações adversas em pacientes oncológicos adultos em uso de imunoterapia no ambiente ambulatorial. Trata-se de uma revisão de escopo, a partir da metodologia JBI. Utilizou-se como estratégia a população dos pacientes oncológicos adultos e idosos em uso de imunoterapia, no conceito das intervenções de enfermagem na administração e controle de reações adversas de imunoterápicos injetáveis, tendo como contexto o ambiente ambulatorial (PCC), em consonância com as diretrizes JBI para revisão de escopo. Foram selecionados 23 artigos, com publicações entre 2005 e 2020. Evidenciou-se três aspectos para discussão: Intervenções de enfermagem em relação a educação em saúde paciente/ família em uso de imunoterápicos, intervenções de enfermagem relacionadas a aplicação dos imunoterápicos no paciente e intervenções relacionadas as reações adversas após a aplicação dos imunoterápicos. É evidente o quanto faz-se necessário o exame físico realizado pelo enfermeiro e o conhecimento farmacológico sobre os imunoterápicos. Sugere-se que a sistematização da assistência de enfermagem seja presente nas instituições de saúde, e que haja elaboração de protocolos institucionais que facilitem a prática profissional de enfermagem em imunoterapia.

Palavras-chave: Imunoterapia; Neoplasia; Enfermagem oncológica.

\begin{abstract}
This study aims to map the scientific evidence on nursing interventions related to the management of adverse reactions in adult oncology patients using immunotherapy in the outpatient setting. This is a scoping review, based on the JBI methodology. The population of adult and elderly oncology patients using immunotherapy was used as a strategy, in the concept of nursing interventions in the administration and control of adverse reactions of injectable immunotherapies, with the outpatient setting (PCC) as the context, in line with the JBI guidelines for scoping review. Twenty-three articles were selected, with publications between 2005 and 2020. Three aspects were evidenced for discussion: Nursing interventions in relation to patient/family health education using immunotherapeutics, nursing interventions related to the application of immunotherapeutics to the patient and related interventions adverse reactions after the application of immunotherapeutics. It is evident how much it is necessary the physical examination performed by the nurse and pharmacological knowledge about immunotherapeutics. It is suggested that the
\end{abstract}


systematization of nursing care is present in health institutions, and that institutional protocols are developed to facilitate the professional nursing practice in immunotherapy.

Keywords: Immunotherapy; Neoplasm; Oncology nursing.

\section{Resumen}

Este estudio tiene como objetivo mapear la evidencia científica sobre las intervenciones de enfermería relacionadas con el manejo de reacciones adversas en pacientes adultos con cáncer que utilizan inmunoterapia en el ámbito ambulatorio. Se trata de una revisión del alcance basada en la metodología JBI. La estrategia utilizada fue la población de pacientes oncológicos adultos y ancianos que utilizan inmunoterapia, en el concepto de intervenciones de enfermería en la administración y control de reacciones adversas de las inmunoterapias inyectables, teniendo como contexto el ambiente ambulatorial (CCP), en línea con las directrices del JBI. para revisión del alcance. Se seleccionaron veintitrés artículos, con publicaciones entre 2005 y 2020. Se evidenciaron tres aspectos para la discusión: Intervenciones de enfermería en relación con la educación en salud del paciente / familia utilizando inmunoterapias, intervenciones de enfermería relacionadas con la aplicación de inmunoterapias en el paciente e intervenciones relacionadas reacciones adversas después de la aplicación de inmunoterapéuticos. Es evidente la necesidad de la exploración física realizada por la enfermera y el conocimiento farmacológico sobre inmunoterapéutica. Se sugiere que la sistematización de los cuidados de enfermería debe estar presente en las instituciones de salud y que se elaboren protocolos institucionales que faciliten la práctica profesional de enfermería en inmunoterapia.

Palabras clave: Inmunoterapia; Neoplasma; Enfermería oncológica.

\section{Introdução}

Câncer é o nome dado a um conjunto de mais de 100 doenças, nas quais possuem em comum a característica do crescimento desordenado de células, podendo invadir tecidos e órgãos vizinhos. Uma célula normal pode sofrer uma mutação genética. Dessa forma o seu material genético alterado passa a receber instruções erradas sobre o desempenho de suas atividades. (INCA, 2020)

A Organização Mundial de Saúde, World Health Organization (WHO) relatou que em 2018 o número de pessoas ao redor do mundo que teve câncer foi de 18,1 milhões. Dessas, 9,6 milhões morreram da doença. A estimativa para 2040 é que esses números podem dobrar.

A estimativa para cada ano do triênio 2020-2022, no Brasil, aponta que ocorrerão 625 mil casos novos de câncer (excluindo pele não melanoma) (World Health Organization, 2020). Apesar da grande evolução no tratamento do câncer, especialmente na abordagem quimioterápica e radioterápica, muitas reações adversas são observadas nos pacientes, pois são terapêuticas que afetam tanto as células cancerígenas quanto as normais. Neste sentido, a busca de ações terapêuticas alvoespecíficas, menos agressivas e com menos eventos adversos, vêm sendo estudadas (Brasil, 2019).

Nos últimos anos a imunoterapia, tem sido amplamente utilizada no tratamento oncológico, dada a sua ação terapêutica frente a inúmeros tipos de tumores. Esse tipo de tratamento baseia-se na utilização do próprio sistema imunológico para induzir uma resposta antitumoral, à partir do reconhecimento de antígenos na superfície do tumor (Cordeiro et al., 2019). Essa resposta ativa linfócitos $\mathrm{T}$, resultando na proliferação e na liberação de citocinas que estimulam a geração de linfócitos T efetores, que ao se infiltrarem, iniciam a destruição do tumor (Cordeiro et al., 2019).

A imunoterapia demanda investigações acerca de seu uso, dada sua especificidade comparada aos demais quimioterápicos, pois o seu foco não são as células tumorais e sim os componentes do sistema imunológico. Dentro desta modalidade, o enfermeiro é o responsável pelo manuseio, administração da droga e ação em quaisquer eventos adversos imunorrelacionados. Observa-se, portanto, o quanto são necessárias intervenções específicas, frente aos possíveis diagnósticos de enfermagem identificados junto ao paciente oncológico em imunoterapia, o que demanda ampliação do conhecimento de toda a equipe de enfermagem, principalmente quanto ao manejo dos eventos adversos (Cordeiro et al., 2019; Wainstein et al., 2017). 
A utilização da imunoterapia para tratamento do câncer traz taxas de resposta variando de $10 \%$ a mais de $50 \%$ em diferentes cenários, bem como a possibilidade de respostas duradouras, trazendo benefício a longo prazo para os que a utilizam. Isso somado ao número crescente de imunoterápicos disponíveis, juntamente com a grande possibilidade de combinações e a expectativa da expansão nas indicações clínicas nos anos por vir (Cordeiro et al., 2019).

Diante do exposto emerge a seguinte pergunta norteadora da pesquisa: quais as intervenções de enfermagem relacionadas ao manejo de reações adversas em pacientes oncológicos em uso de imunoterapia no ambiente ambulatorial?

Foi realizada uma pesquisa preliminar no PROSPERO, Cochrane Sitematic Review, Open Science Framework e na Medline buscando protocolos e estudos de revisão em andamento sobre a temática. Foi constatada então, uma ausência de protocolos construídos com a temática que falassem a respeito das intervenções de enfermagem e os imunoterápicos.

Desse modo, construiu-se um protocolo para revisão de escopo a partir do JBI SUMARI, tendo como objetivo: mapear as evidências científicas sobre intervenções de enfermagem relacionadas ao manejo de reações adversas em pacientes oncológicos adultos em uso de imunoterapia no ambiente ambulatorial.

\section{Metodologia}

\subsection{Tipo de estudo}

Trata-se de uma revisão de escopo conduzida a partir do modelo o Joanna Briggs Institute (JBI), conforme o Manual para Revisão de escopo JBI (Bulechek, 2011), e a construção do artigo seguiu o Check-list PRISMA-SCR, específico para construção de revisões de escopo.

\subsection{Estratégia para coleta de dados}

Para formulação da questão de pesquisa foi empregada a estratégia PCC (População: pacientes oncológicos adultos e idosos em uso de imunoterapia/ Conceito: intervenções de enfermagem na administração e controle de reações adversas de imunoterápicos injetáveis/ Contexto: no ambiente ambulatorial), em consonância com as diretrizes JBI para revisão de escopo, que gerou a questão de investigação: "quais as intervenções de enfermagem relacionadas ao manejo de reações adversas em pacientes oncológicos em uso de imunoterapia no ambiente ambulatorial?"

Para considerar a população foi utilizado o critério de classificação da faixa etária preconizado pela OMS, na qual considera-se adultos indivíduos entre 18 e 65 anos e idosos a partir de 65 anos; sem distinção do tipo de câncer e que estivessem utilizando qualquer medicamento considerado imunoterápico por via endovenosa.

Sobre o conceito da estratégia PCC, leva-se em consideração os cuidados específicos da equipe de enfermagem nas reações adversas durante o tratamento desses pacientes.

Considerou-se apenas artigos que retratassem esses cuidados no âmbito ambulatorial, no qual o paciente não necessita estar internado em rede hospitalar para realizar seu tratamento.

\subsection{Estratégias de busca}

Foi realizada uma busca prévia nas bases de dados: Medline/Pubmed e Cinahl/Ebsco. Para essa busca prévia foram utilizados os descritores Mesh: Immunotherapy, Oncology nursing e Neoplasms, usando operador booleanos AND para todos os termos. Dessa forma a equação de busca utilizada foi "Immunotherapy" AND "Oncology nursing" AND "Neoplasms".

Em seguida, ampliou-se a busca nas bases de dados: Embase e o portal de periódicos Scielo, adaptando os descritores em cada base, ficando da seguinte forma: "Immunotherapy" and "Oncology nursing" and "Neoplasms" para a Embase, totalizando 235 referências recuperadas e sete selecionadas e "Enfermagem" and "tratamento" and "neoplasia", na qual recuperou-se 23 artigos e foram selecionados dois para a base de dados Scielo. Buscou-se em seguida no Google scholar com 
as palavras-chave: imunoterapia e intervenções de enfermagem, na qual emergiu 633 artigos referentes à literatura cinzenta. Desses 633 cinco foram selecionados para leitura na íntegra e apenas um foi incluído. O período de busca e seleção compreendeu os meses de janeiro e fevereiro de 2021.

Foi construído um fluxo para descrever a análise das referências identificadas nas bases de dados, demonstrado na Figura 1.

Figura 1: Fluxo das análises das referências.
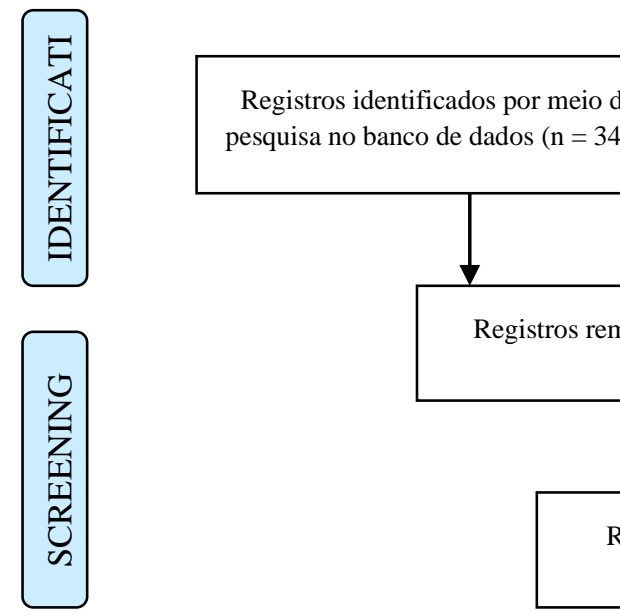
pesquisa no banco de dados $(n=343$

Registros adicionais por outras fontes (google acadêmico $(n=633)$
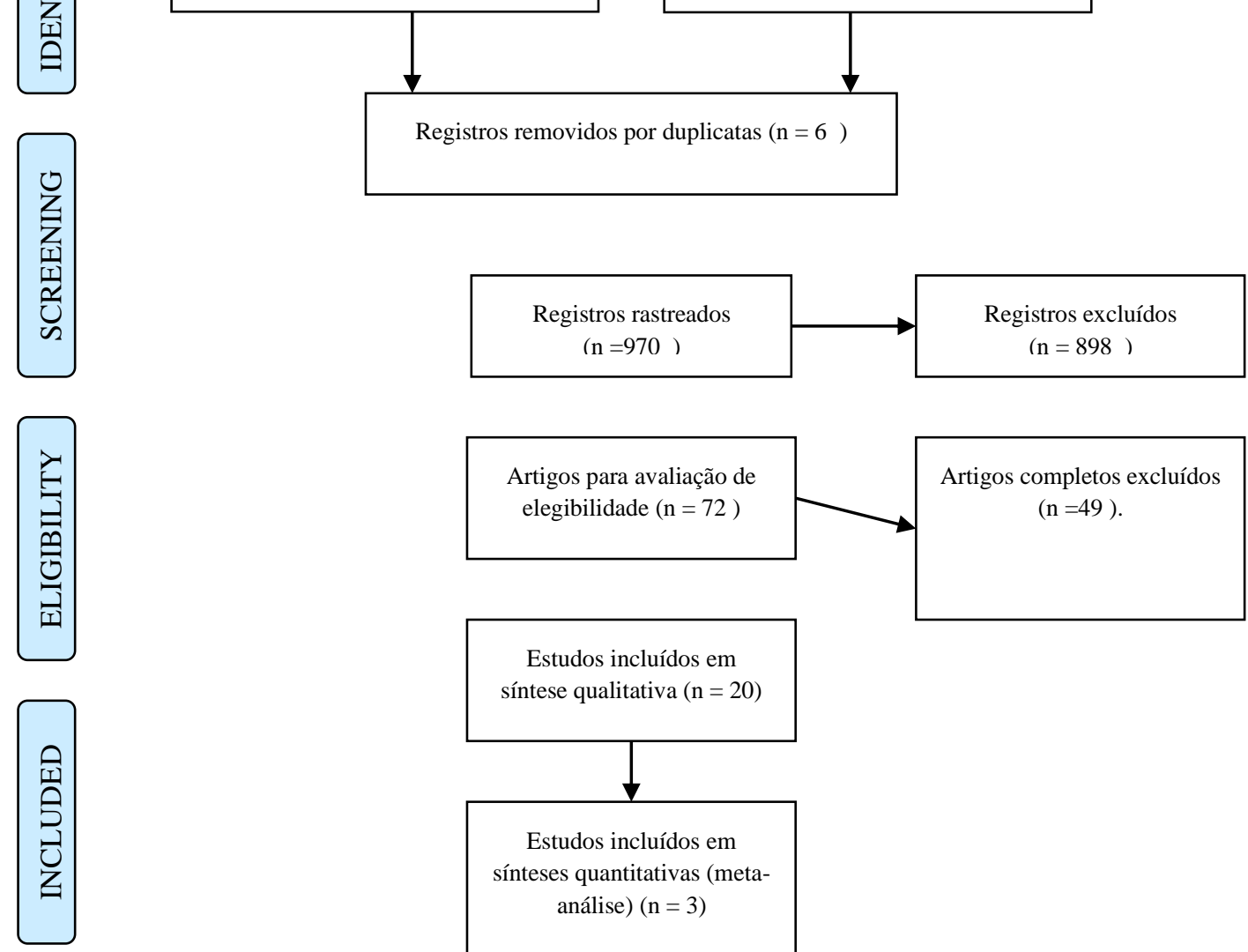

Estudos incluídos em síntese qualitativa $(\mathrm{n}=20)$

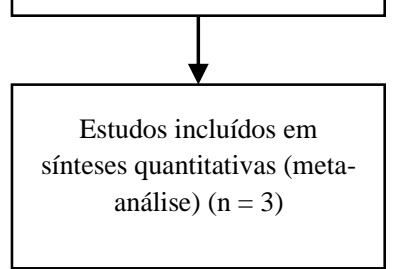

Fonte: Dados da Revisão.

\subsection{Critérios de seleção}

Critérios de inclusão: faixa etária: adultos (acima de 18 a 64 anos) e idosos (acima de 65); idiomas: inglês, português e espanhol, publicados no período de 2005 à 2021.

Critérios de exclusão: estudos com pacientes internados que faziam uso de quimioterápicos de modo concomitante a imunoterapia e em uso de imunoterápicos orais que apresentam intervenções diferentes das drogas injetáveis.

\subsection{Seleção dos estudos}

Os artigos recuperados foram organizados na Plataforma de organização de referências Mendeley. Sendo o mesmo programa utilizado para remoção das duplicatas. A seleção dos artigos foi realizada por dois revisores independentes a partir da leitura primeiramente dos títulos e resumos, e posteriormente a leitura dos textos completos para extração dos dados. 


\subsection{Análise e tratamento dos dados}

Os estudos relevantes foram recuperados na íntegra e os seus dados de citação foram importados para o JBI SUMARI. Nos casos em que houve divergência entre os revisores foi solicitado parecer de um terceiro revisor com maior experiencia na área de imunoterápicos.

Foi estruturado um formulário específico para facilitar e sistematizar a extração dos dados pelos revisores, alinhados à pergunta e objetivo dessa pesquisa.

Os dados extraídos foram apresentados em forma de tabelas contendo: autores, ano de publicação, local, objetivo, população, métodos e principais resultados referentes às intervenções de enfermagem nas reações adversas apresentadas nos pacientes oncológicos em uso de imunoterapia, alinhado ao objetivo desta revisão de escopo.

Assim, cumprida esta etapa, os dados extraídos foram compilados e organizadas em três aspectos: Intervenções de enfermagem em relação a educação em saúde paciente/ família ao uso de imunoterápicos, intervenções de enfermagem relacionadas a aplicação dos imunoterápicos no paciente e intervenções relacionadas as reações adversas após a aplicação dos imunoterápicos.

\subsection{Aspectos éticos}

Por tratar-se de uma revisão de escopo não foi necessário submissão à comitê de ética.

Quanto aos pesquisadores que redigiram o estudo, também não houve conflitos de interesse ou problemas éticos, estando de comum acordo para construção e publicação do artigo.

\section{Resultados}

Conforme resultado das buscas, obteve-se a construção da seguinte tabela demonstrada abaixo: 
Tabela 1: Distribuição de artigos localizados nos Portais/Bases de dados e composição da amostra - Rio de Janeiro, RJ, Brasil, 2007/2020.

\begin{tabular}{|c|c|c|c|c|c|c|}
\hline $\begin{array}{c}\text { Portais/Bases de } \\
\text { Dados }\end{array}$ & Equação de busca & Registros Recuperados & $\begin{array}{c}\text { Registros após exclusão } \\
\text { de duplicatas }\end{array}$ & $\begin{array}{l}\text { Número de registros } \\
\text { excluídos }\end{array}$ & $\begin{array}{c}\text { Número de } \\
\text { registros após } \\
\text { aplicação dos critérios }\end{array}$ & Amostra \\
\hline MEDLINE/ PUBMED & $\begin{array}{l}\text { Immunotherapy and } \\
\text { Oncology nursing and } \\
\text { Neoplasms }\end{array}$ & 40 & 40 & 21 & 19 & 8 \\
\hline MEDLINE/ EBSCO & $\begin{array}{l}\text { Oncology nursing and } \\
\text { Antineoplastic Protocols } \\
\text { and Immunotherapy }\end{array}$ & 3 & 3 & 1 & 2 & 2 \\
\hline CINAHL/ EBSCO & $\begin{array}{l}\text { Oncology nursing and } \\
\text { Immunotherapy }\end{array}$ & 29 & 29 & 19 & 10 & 3 \\
\hline SCIELO & $\begin{array}{c}\text { Enfermagem } \\
\text { and } \\
\text { tratamento } \\
\text { and neoplasia }\end{array}$ & 23 & 23 & 21 & 2 & 2 \\
\hline EMBASE & $\begin{array}{l}\text { Immunotherapy and } \\
\text { Oncology nursing and } \\
\text { Neoplasms }\end{array}$ & 235 & 234 & 208 & 26 & 7 \\
\hline $\begin{array}{c}\text { GOOGLE } \\
\text { ACADÊMICO }\end{array}$ & $\begin{array}{l}\text { Imunoterapia e } \\
\text { intervenções de } \\
\text { enfermagem }\end{array}$ & 633 & 628 & 623 & 5 & 1 \\
\hline TOTAL & & 976 & 6 & 898 & 72 & 23 \\
\hline
\end{tabular}

Fonte: Autores. 
Os 23 estudos selecionados compreendiam publicações do período de 2007 até o ano de 2020. Da metodologia utilizada na pesquisa havia um percentual majoritário de estudos qualitativos $(n=19)$ e editoriais de revista $(n=1)$ e demais estudos quantitativos em menor percentual $(n=3)$.

Os resultados das intervenções localizadas na coleta de dados, são apresentados no Quadro1:

Quadro 1: Distribuição da amostra por categorias e resultados das intervenções de enfermagem - Rio de Janeiro, RJ, Brasil, $2007 / 2020$

\begin{tabular}{|c|c|c|}
\hline Cate & Referências & Intervenções no manejo de reações adversas em pacientes oncológicos em uso de imunoterapia \\
\hline $\begin{array}{l}\text { Intervenções de } \\
\text { enfermagem em } \\
\text { relação a a } \\
\text { educação em } \\
\text { saúde paciente/ } \\
\text { família ao uso } \\
\text { de } \\
\text { imunoterápicos }\end{array}$ & 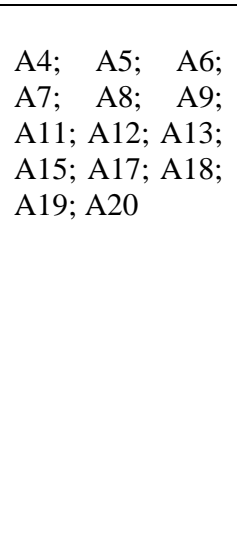 & $\begin{array}{l}\checkmark \\
\text { A equipe de enfermagem é especialista em gerenciamento de sintomas, educação do } \\
\text { paciente e cuidados de suporte para pacientes com câncer e seus familiares e cuidadores; } \\
\checkmark \quad \text { Educar cuidadosamente os pacientes e famílias e cuidadores para promover adesão aos } \\
\text { cuidados recomendados e apoiar a tomada de decisão; } \\
\checkmark \quad \text { Importante educar e informar os médicos sobre como identificar, classificar e gerenciar } \\
\text { essas toxicidades imunomediadas; } \\
\checkmark \quad \text { A comunicação deve ser destacada ao desenvolver um plano de tratamento com o paciente e } \\
\text { cuidador. Pacientes devem se sentir confortáveis em alcançar a equipe de oncologia com qualquer } \\
\text { dúvida ou preocupações relacionadas ao tratamento, e os pacientes e cuidadores devem compreender a } \\
\text { importância de relatar qualquer novo ou sintomas de mudança; } \\
\checkmark \quad \text { Tomada de decisão compartilhada são importantes ao planejar as opções de tratamento; } \\
\checkmark \quad \text { Compreender o mecanismo de ação ajuda a determinar o risco potencial para os pacientes, } \\
\text { seus familiares, e profissionais de saúde; } \\
\checkmark \quad \text { Enfermeiros têm um papel crucial no fornecimento de pacientes e cuidadores educação } \\
\text { sobre as informações de segurança pertinentes, incluindo a incidência e sintomas de irAEs; }\end{array}$ \\
\hline $\begin{array}{l}\text { Intervenções de } \\
\text { enfermagem } \\
\text { relacionadas a } \\
\text { aplicação dos } \\
\text { imunoterápicos } \\
\text { no paciente }\end{array}$ & $\begin{array}{l}\text { A2; A10; A11; } \\
\text { A12; A15; A16; } \\
\text { A18 }\end{array}$ & $\begin{array}{l}\checkmark \\
\checkmark \\
\text { tempos de infusão variam de } 30 \text { a } 60 \text { minutos; } \\
\checkmark \quad \text { Atenção para administração: a maioria das imunoterapias são dosados em duas ou três } \\
\text { semanas com ciclos a cada } 14 \text { ou } 21 \text {, com infusões de } 30 \text { - } 60 \text { minutos. } \\
\checkmark \quad \text { A aplicação desses padrões à administração de imunoterapia garante que verificações } \\
\text { independentes entre dois profissionais considerados competentes na administração de imunoterapia } \\
\text { estão verificando e documentando componentes críticos de administração pedidos, como } \\
\text { identificadores de pacientes, medicamentos nome e dose, via e taxa de administração e variáveis de } \\
\text { cálculo de dosagem; } \\
\checkmark \quad \text { Medir e interpretar os sinais vitais correspondentes às horas } 0 \text { e } 2 \text { horas de imunização; } \\
\checkmark \quad \text { Doses imunoterápicas são administradas por completo; } \\
\checkmark \quad \text { Nenhum outro medicamento deve ser co-administrado usando a mesma linha de infusão }\end{array}$ \\
\hline $\begin{array}{l}\text { Intervenções } \\
\text { relacionadas as } \\
\text { reações } \\
\text { adversas após a } \\
\text { aplicação dos } \\
\text { imunoterápicos. }\end{array}$ & $\begin{array}{l}\text { A1; A4; A5; } \\
\text { A6; A7; A8; } \\
\text { A9; A11; A12; } \\
\text { A13; A15; A17; } \\
\text { A18; A19; A20 }\end{array}$ & $\begin{array}{l}\checkmark \\
\text { Tratamento de erupção relacionada ao sistema imunológico consiste em esteróides tópicos, } \\
\text { emolientes, anti-histamínicos para eventos adversos de grau } 1 \text { e } 2 \text {, enquanto a terapia com o inibidor } \\
\text { pode ser continuada; } \\
\checkmark \quad \text { Efeitos colaterais moderados a graves (graus } 3 \text { a } 5 \text { ), que podem ser sérios e com risco de } \\
\text { vida, exigem esteróides mais agressivos terapia e diminuição gradual da dosagem de pelo menos um } \\
\text { mês ou descontinuar a terapia; } \\
\checkmark \quad \text { É comum os pacientes sofrerem colite ou diarreia pelos imunoterápicos. Fazer registros e } \\
\text { acompanhamento; } \\
\checkmark \quad \text { Toxicidade dermatológica: prurido, rush cutâneo, vitiligo, rush maco-papular, pápulas e } \\
\text { ulceração (uso de corticoide ou interrompimento da imunoterapia) } \\
\checkmark \quad \text { A diferenciação e intervenção precoce de irAEs são importantes para evitar piora dos } \\
\text { sintomas e possíveis alterações na terapia planejada; } \\
\checkmark \quad \text { Reconhecimento imediato e intervenção precoce de irAEs é a estratégia de gestão mais } \\
\text { eficaz; } \\
\checkmark \\
\text { com CTCAE; } \\
\checkmark \\
\text { profissionais de enfermagem devem incorporar um monitoramento e gerenciamento desses irAEs; }\end{array}$ \\
\hline
\end{tabular}

Fonte: Autores. 


\section{Discussão}

\subsection{Intervenções de Enfermagem em relação a educação em saúde paciente/ família}

A imunoterapia por ser uma terapêutica recente quando comparada a quimioterapia convencional, exige uma boa formação da equipe de enfermagem sobre os mecanismos fisiopatológicos e manejos de sinais e sintomas (Cordeiro et al., 2019).

A imunoterapia aciona a produção de anticorpos que irão combater o tumor. Apesar de ser mais especifica por ser uma reação antígeno-anticorpo, também pela exacerbação do sistema imunológico, pode causar reações de alta resposta, ou seja, o número alto de anticorpos, podem sobrecarregar órgãos vitais, como pulmão e o fígado (Bulechek, 2011).

Outra informação é sobre o uso de medicações não prescritas, durante o tratamento. As medicações, como corticoides, só podem ser ingeridas com prescrição médica, porque são fármacos depressores do sistema de resposta imunológico e por isso, diminuem a eficácia do imunoterápico (The Joanna Briggs Institute, 2015).

Desse modo, o conhecimento prévio sobre como será o tratamento e a forma de aplicação do imunoterápico, torna o tratamento mais seguro e tranquilo para os pacientes e familiares (Bruneto et al., 2019; Gordon et al., 2017). Sugere-se que na consulta de enfermagem siga as seguintes recomendações:

1-Orientar sobre os mecanismos de ação das drogas, de forma clara e compreensível (The Joanna Briggs Institute, 2015);

2- Orientar a não ingerir medicações não prescritas pelo oncologista, considerando a existência de drogas prejudiciais como: antialérgicos e corticóides (Serra et al., 2019; Galioto \& Mucenski, 2019);

3-Instruir quanto os efeitos colaterais da droga como: dor local, cefaleia, distúrbios hepático, gastrointestinais e pulmonares (The Joanna Briggs Institute, 2015).

4- Explicar ao paciente sobre o tratamento e suas etapas, destacando o uso da pré-medicação quando necessário. Vale citar as medicações, mais utilizadas durante o tratamento para evitar episódios de reações anafilaxia (Hidrocortisona; Difenidramina, metilprednisolona) e antitérmicos como paracetamol (Bruneto et al., 2019);

5- Educar sobre importância da periodicidade das sessões e a busca da equipe de saúde (The Joanna Briggs Institute, 2015);

6- Incluir a família no cuidado ao paciente (Becze, 2015).

Destaca-se a importância da construção de um vínculo entre equipe, paciente e família, o que contribui para a minimização do abandono das sessões proporcionando um aumento da taxa de sucesso terapêutico (Serra et al., 2019; Bartell et al., 2015).

Ainda, a consulta de enfermagem, fundamentada no Processo de Enfermagem (PE), é um momento legal e de grande ajuda para formação do vínculo cliente-profissional. Um dos objetivos dessas ações é promover a integração, contribuindo para a qualidade da assistência prestada. A implementação da consulta deve ser valorizada e implementada nas unidades de saúde, principalmente quando se cuida de clientes oncológicos (Wiley et al., 2017).

As informações podem se impressas e oferecidas por meio de um livreto educacional, desenvolvido especificamente pela enfermeira, trazendo recomendações a serem seguida pela equipe (The Joanna Briggs Institute, 2015). Ainda, a inserção da tecnologia é uma ferramenta que possibilita o acompanhamento via telefone celular do cliente mesmo a distância (Seery, 2017). 


\subsection{Intervenções de enfermagem relacionadas a administração do imunoterápicos}

A imunoterapia visa usar o sistema imunológico para combater o câncer. A resposta induzida pelos imunoterápicos são: fase de eliminação (ativa-se a resposta imune inata e adotiva culminando com a destruição de células tumorais), fase de equilíbrio (nesta etapa células tumorais podem sobreviver ao ataque do sistema imunológico, as células sobreviventes sofrem processo de modificação) e a Fase de escape (células tumorais variantes e editadas começam a proliferar e a estabelecer um ambiente tumoral) (Sheldon, 2016).

Os imunoterápicos mais comuns são: Vacinas anticâncer, Terapia com receptor de antígeno quimérico de célula T (CAR T cell therapy) e Terapias com bloqueio de checkpoint imunológico (imune checkpoint blockade therapy). Anticorpos monoclonais são versões de proteínas do sistema imune que se ligam em regiões específicas a célula tumoral. Os Inibidores Checkpoint são drogas que estimulam fortemente o sistema imunológico, o que ajuda a reconhecer e atacar as células cancerígenas. Já as vacinas são substâncias que estimulam o sistema imune a iniciar uma resposta à certas doenças (Sheldon, 2016).

Os protocolos da imunoterapia são realizados em ciclos, administradas em intervalos de duas ou três semanas, com tempo de administração entre 30 a 60 minutos, dependendo da medicação (The Joanna Briggs Institute, 2015).

Os imunoterápicos como Ipilimumabe, Nivolumabe e Pembrolizumabe, não requerem pré-medicação. Embora, alguns farmacologistas ainda solicitem acetaminofeno, difenidramina ou famotidina, antes da administração (Tolentino et al., 2016). Para evitar falha na terapêutica sempre seguir o protocolo de cada instituição.

Destaca-se a importância de reconhecimento da composição das medicações que serão administradas, a dosagem adequada ao paciente e a checagem antes da administração. Recomenda-se conferência da prescrição, quanto nome do paciente, matrícula, nome da droga, dosagem, via de infusão e tempo de administração (Ysebaert et al., 2019)

A equipe de enfermagem tem papel fundamental no manejo dos imunoterápicos (Silvestrini, 2020). O registro e checagem das medicações são obrigatórias para o enfermeiro responsável pela administração da imunoterapia, com nome, data e horário e dose tanto em prontuário físico como no eletrônico, sendo carimbado e assinado de forma legível. Esta conduta deve ser padrão em todas as unidades que trabalhem com imunoterápicos (Becze, 2016; Gallegos et al., 2019; Madden \& Hoffner, 2017).

A anamnese realizada pelos enfermeiros precisa incluir avaliações psiquiátricas com perguntas sobre alterações de humor e no padrão de sono secundários ao hipotireoidismo ou hipertireoidismo induzidos por drogas imunoterápicos e, nas avaliações físicas, com exame físico completo, incluindo queixas relatadas, monitoramento do peso, dor abdominal, fadiga e dor local da aplicação. E realização monitoramento após a administração, incluindo toxicidade e complicações (Gallegos et al., 2019).

\subsection{Intervenções relacionadas as reações adversas após a aplicação}

Reação adversa a medicamento é caracterizada por ser qualquer resposta a um fármaco que seja prejudicial, ocorrendo em doses normalmente utilizadas em seres humanos para profilaxia, diagnóstico e tratamento de doenças, não intencional. Dessa forma a farmacovigilância avalia sistematicamente essas reações adversas, identifica precocemente a gravidade e as interações não descritas em bula ou na literatura, bem como aumento na frequência das reações conhecidas, os fatores de risco e possíveis mecanismos subjacentes às reações e os sinais de alerta que evidenciam uma relação de causalidade entre fármaco e reação adversa a medicamentos. Tais ações promovem a segurança e o uso racional dos medicamentos (Rubin, 2015).

Qualquer reação adversa deve ser notificada. Para medicamentos comercializados há mais de cinco anos, há necessidade de notificação de qualquer suspeita (Madden \& Hoffner, 2017). 
Na quimioterapia as substâncias químicas, que podem ser únicas ou em combinação, atuam nas células interferindo no seu processo de crescimento e divisão. Suas principais toxicidades são a mielossupressão, náuseas e vômitos, fadiga, alopecia, alterações na motilidade gastrointestinal, perda do apetite e mucosite (Oliveira et al., 2019).

As reações adversas relacionadas a imunoterapia diferem das relacionadas à quimioterapia antineoplásica, visto que estão ligadas à estimulação do sistema imunológico, com ação agente-específico.

Dentre as reações mais comuns estão descritas: dor local, reação cutâneas de leve a grave, hepatite autoimune, distúrbio gastrointestinais, cefaleia e tonturas, toxicidade endócrina e toxicidade pulmonar (Bulechek, 2011; Davies \& Duffield, 2020).

Os eventos adversos musculoesqueléticos são a artralgia e/ou a mialgia. São mais comuns em inibidores checkpoint PD1, quando comparados com inibidores de CTLA-4. A abordagem multidisciplinar é importante no gerenciamento dos eventos musculoesqueléticos. O paciente precisará iniciar anti-inflamatórios e analgésicos, de acordo com o grau da dor, e em caso de sequela ou limitação serão encaminhados a fisioterapia (Sociedade Brasileira de Farmacêuticos em Oncologia \& Agência Nacional de Vigilância Sanitária, 2011). Assim, cabe ao enfermeiro avaliar a dor, medicar de acordo com prescrição, realizar exame físico, com avaliação limitação de movimento e marcha e atuar junto com equipe multidisciplinar (psicólogos, fisioterapeuta, médicos) para controle de sintomas e reabilitação (Wainstein et al., 2017; Moretto et al., 2019).

No que se refere às manifestações dermatológicas, ressaltam-se as características morfológicas, clínicas e histológicas específicas das mesmas. É importante buscar uma correlação clínico-patológica, na qual inclua a história detalhada do tratamento, a aparência clínica das lesões e as características imunopatológicas, a fim de que se tenha um diagnóstico específico, a prevenção de agravos, um tratamento adequado e a possibilidade da continuidade no tratamento (Thomas, 2016).

Dentre as reações adversas causada pelo imunoterápico, está a hepatite autoimune. A Hepatite autoimune, é causada quando os anticorpos passam a atacar os hepatócitos saudáveis, causando uma inflamação, que se não tratada precocemente podem vir a evoluir para um quadro de cirrose hepática. O tratamento para essa morbidade é o corticoide terapia, e as vezes é necessário interromper as sessões. Cabe aos enfermeiros no exame físico do paciente avaliar a região abdominal, quanto distensão e dor a palpação profunda e as mucosas em busca de sinais de icterícia. A hepatite autoimune pode ser controlada com administração de corticoides, mas necessita de detecção precoce (Bulechek, 2011).

A neurotoxicidade é causada pela liberação de um número elevado de citocinas relacionados ao sistema imunoterapêuticos denominado Cart $\mathrm{T}$ cell. Com a elevada toxicidade por conta das citocinas, o paciente pode apresentar cefaleia, tontura e alteração motora (Abdel-Rahman et al., 2017). As condutas do enfermeiro são ouvir e registrar queixas como: cefaleias, quanto intensidade e periodicidade, a avaliação cognitiva, incluindo nível de consciência, presença de Dellirium e perda da memória. Em caso, positivo de algum desses sintomas, encaminhar para avaliação médica (Moretto et al., 2019).

Os distúrbios gastrointestinais são frequentes durante a imunoterapia. Os sintomas mais presentes são: a diarreia, dor abdominal e hematoquezia acompanhada de febre. Devem ser investigados diagnósticos diferenciais, como infecções bacterianas e virais através de avaliação de exames de fezes simples e para investigar a presença de Clostridium difficile. Pode se evoluir para um quadro de Colite, caracterizada por sinais de inflamação intestinal, diagnosticados em colonoscopia. Embora menos comum, é considerada como evento de maior gravidade, podendo resultar na descontinuidade do tratamento. Nesses casos as intervenções de enfermagem são: hidratação oral e endovenosa se necessários, corticoide (dose ajustada de acordo com gravidade), exame de fezes e hidratação endovenosa se necessária (Becze, 2016; Orue et al., 2007).

A pneumonite é uma complicação pouco frequente, com incidência estimada em cerca de 4\%, mas trata-se de uma intercorrência de difícil diagnóstico e com grande morbimortalidade. É dividida em cinco graus (1- assintomático, 2sintomático com limitações da atividade diária, - sintomático com limitações do autocuidado ou hipóxia, grau 4 sintomático 
com limitações do autocuidado e alto risco a vida e grau 5 - iminência de morte), sendo que o último pode levar ao óbito. As inflamações nas células pulmonares são detectadas através de exame clínico e radiológico, seja por raio-x ou tomografia. As intervenções para essa reação adversa são: monitorização dos sinais vitais e oximetria, administrar corticoide endovenoso e oferecer oxigenoterapia, quando prescritos. Quanto antes diagnosticado, melhor o prognóstico (Garrett, 2019).

A toxicidade endócrina manifesta-se quando ação dos anticorpos atinge a hipófise, tireoide, glândulas adrenais e pâncreas. A hipofisite e o hipotireoidismo são as manifestações mais frequentes, mas existem os casos de hipertireoidismo, insuficiência adrenal e diabetes mellitus tipos 1 e 2. As manifestações clínicas da toxicidade endócrina incluem: astenia, cefaleia, vertigem, tontura, náuseas, vômitos, dor abdominal, alopecia, alterações autonômicas, comportamentais, visuais, ponderais, do apetite e do ritmo intestinal (Gallegos et al., 2019; American Cancer Society, 2020; Franco \& Natal, 2018). Como intervenções de enfermagem destacam-se: a avaliação da glicemia capilar, a avaliação da dosagem de exame laboratoriais dos hormônios tireoidianos (T3, T4, TSH) e a avaliação da densidade urinária (se apresentar poliúria) e orientar quanto a reposição hormonal, se caso prescrita pela equipe médica.

A identificação precoce pelo enfermeiro de qualquer sintoma de reação adversa e a rápida intervenção é de fundamental importância para evitar que haja agravos ao paciente. Observa-se um consenso da importância das intervenções de enfermagem junto ao paciente e a família. O cuidado de enfermagem é primordial no tratamento com imunoterápicos, porque são medicamentos de ação complexa no organismo e necessitam de atenção especializada, bem capacitada tanto para educar, administrar e prevenir as complicações clínicas (Helber et al., 2018; Vazquez, 2017).

\section{Conclusão}

A expansão do uso da imunoterapia para tratamento do câncer, traz consigo a necessidade de maior aprimoramento sobre as intervenções de enfermagem e a aplicação na prática assistencial. As intervenções que mais se destacaram foram aquelas relacionadas à educação ao paciente/família e às reações adversas. Com relação às intervenções relacionadas com a administração do imunoterápico, destaca-se a relevância do exame físico realizado pelo enfermeiro e o conhecimento técnico científico sobre a farmacologia das drogas imunoterápicas.

A quantidade expressiva de eventos adversos relacionados a imunoterapia demonstra que a terapêutica requer uma atenção especial.

O estudo teve por limitação o diminuto acervo de publicações acerca das intervenções de enfermagem em imunoterapia aplicáveis à realidade brasileira.

Diante do exposto, existe uma necessidade de expandir os estudos nacionais na área da imunoterapia e sugere-se que o processo de enfermagem seja utilizado como base na elaboração de protocolos institucionais que facilitem a prática profissional de enfermagem em imunoterapia.

\section{Referências}

Abdel-Rahman, O., Eltobgy, M., Oweira, H., Giryes, A., Tekbas, A., \& Decker, M. (2017). Immune-related musculoskeletal toxicities among cancer patients treated with immune checkpoint inhibitors: a systematic review. Immunotherapy, 9(14), 1175-1183.

American Cancer Society. (2020). CAR T-cell therapy and its side effects. ACS.

Bartell, H., Wolchok, J., Hodi, F. S., Liu, H., Wojtaszek, C., \& Weber, J. (2015). Immuno-oncology safety education experience: key lessons from ipilimumab (IPI). Journal for ImmunoTherapy of Cancer, 3(2), P384.

Becze, E. (2015). Immunotherapy experts identify key oncology nursing priorities ons summit. ONS VOICE.

Becze, E. (2016). An oncology nursing overview of new immune checkpoint inhibitors. ONS VOICE.

Brasil. (2019). Estimativa 2020: incidência de câncer no Brasil. INCA. 
Brasil. (2020). ABC do câncer: abordagens básicas para o controle do câncer. INCA.

Bruneto, R. V., Arruda, G. N., Fernandes, K. T., Pacca, F. C., \& Veiga, T. (2019). Anticorpos monoclonais no tratamento oncológico: revisão de literatura para o atendimento ao paciente e manejo das reações infusionais. Archives of Health Sciences, 26(3), 173-178.

Bulechek, G. (2011). Classificação das Intervenções de Enfermagem (NIC). Elsevier Health Sciences.

The Joanna Briggs Institute. (2015). The Joanna Briggs Institute Reviewers'Manual 2015: Methodology for JBI Scoping Reviews. JBI.

Cordeiro, M. L. S., Silva, N. L. F., Vaz, M. R. F., \& Nóbrega, F. F. F. (2014). Anticorpos monoclonais: implicações terapêuticas no câncer. Revista Saúde \& Ciência Online, 3(3), 253-265.

Davies, M., \& Duffield, E. (2020). Durvalumab immunotherapy: nursing management of immune-related adverse events during the journey of patients with stage III non-small cell lung cancer. Clinical Journal of Oncology Nursing, 24(3), 277-283.

Franco, A. O., \& Natal, S. L.(2018). Eventos adversos imunorrelacionados e seu manejo na imunoterapia em pacientes oncológicos. Revista Oswaldo Cruz, $5(19)$

Galioto, M., \& Mucenski, J. (2019). Immunotherapy summit: proceedings and identified priorities for safe administration and care. Clinical Journal of Oncology Nursing, 23(3), E60-E65.

Gallegos, R., Kogelman, A., Wagner, M., Cloud, A., Olson, M., Robideau, K., Patrick, L., Comfort, J., \& Hirko, K. (2019). Chemotherapy Education: an interprofessional approach to standardizing processes and improving nurse and patient satisfaction. Clinical Journal of Oncology Nursing, 23 (3), 309-314.

Garrett, N. F. M. S. (2019). Prevalência de toxicidades dermatológicas em pacientes com câncer submetidos ao tratamento com imunoterápicos: revisão sistemática e metanálise. Dissertação de Mestrado, Universidade de Brasília.

Gordon, R., Kasler, M. K., Stasi, K., Shames, Y., Errante, M., Ciccolini, K., Lucas, A. S., Raasch, P., \& Fischer-Cartlidge, E. (2017). Checkpoint inhibitors: common immune - related toxicities. Clinical Journal of Oncology Nursing, 21(2), 45-52.

Helber, H. A., Hada, A. L., Pio, R. B., Moraes, P. H. Z., \& Gomes, D. B. D. (2018). Immunotherapy-induced pneumonitis: cases report. Jornal Einstein (Sao Paulo), 16(2), eRC4030.

Madden, K. M., \& Hoffner, B. (2017). Ipillimumab-Based therapy: consensus statement from the faculty of the melanoma nursing initiative on managing adverse events with ipilimumab and combination therapy with nivolumab. Clinical Journal of Oncology Nursing, 21(4), 30-41.

Moretto, I. G., Contim, C. L. V., \& Santo, F. H. E. (2019). Acompanhamento por telefone como intervenção de enfermagem a pacientes em quimioterapia ambulatorial. Revista Gaúcha de Enfermagem, 40, e20190039.

Oliveira, P. P., Bezerril, M. S., Paiva, R. M., Santos, V. E. P., Andrade, F. B., \& Silveira, E. A. A. (2019). Segurança do paciente na administração de quimioterapia antineoplásica e imunoterápicos para tratamento oncológico: scoping review. Revista Texto \& Contexto - Enfermagem, $28,1-18$.

Orue, I. T., Hernández, A. I. J., \& Garcet, M. A. C. (2007). Experiencia de enfermería en la inmunoterapia con gangliósidos en pacientes con cáncer avanzado. Revista Cubana de Enfermagem, 23(3), 1-8.

Rubin, K. M. (2015). Understanding immune checkpoin inhibitors for effective patient care. Clinical Journal of Oncology Nursing, $19(6), 709-717$.

Seery, V. (2017). Interprofessional collaboration with immune checkpoint inhibitor therapy: the roles of gastroenterology, endocrinology and neurology. Seminars in Oncology Nursing, 33(4), 402-414.

Serra, J., Majem, M., Sullivan, I. G., Riudavets, M., Anguera, G., Barba, A., González, I., \& Guàrdia, N. (2019). P1.07-09 implementation of a nursing program for cancer patients treated with immunotherapy by an Immunotherapy Nurse Specialist. Journal of Thoracic Oncology, 14(10), S490-S491.

Sheldon, L. K. (2016). Oncology nurses and the cancer moonshot 2020. Clinical Journal of Oncology Nursing, 20 (4), $355-356$.

Silvestrini, A. A. (2020). Diretrizes oncológicas (3a ed). Diretrizes Oncológicas.

Sociedade Brasileira de Farmacêuticos em Oncologia \& Agência Nacional de Vigilância Sanitária. (2011). Guia para Notificação de Reações Adversas em Oncologia (2a ed). São Paulo: SOBRAFO/ ANVISA. https://sobrafo.org.br/wp-content/uploads/2018/12/ATT00373.pdf

Thomas R. (2016). Understanding immunotherapy for the treatment of non-small cell lung cancer. Brazilian Journal of Nursing, 25 (16), S12-s17.

Tolentino, G. S., Bettencourt, A. R. C., \& Fonseca, S. M. (2019). Construction and validation of an instrument for nursing consultation in outpatient chemotherapy. Revista Brasileira de Enfermagem, 72(2), 391-399.

Vazquez, A. (2017). Hypophysitis Nursing management of immune-related adverse events. Clinical Journal of Oncology Nursing, 21(2), 154-156.

Wainstein, A. J., Calabrich, A., Melo, A. C., Buzaid, A. C., Katz, A., Anjos, C. A., Ferreira, C. G., Baldotto, C., Mathias, C. M. C., Yen, C. T., Sternberg, C., E. Gomes, E., Moura, F., Castro Junior, G., Fernandes, G. S., Hoff, P. M. G., Schmerling, R., Munhoz, R. R., Barroso-Sousa, R., Azevedo, S. J., \& Lima, V. C. C. (2017). Diretrizes brasileiras de manejo de toxicidades imunomediadas associadas ao uso de bloqueadores de correceptores imunes. Brazilian Journal of Oncology, 13(43), 1-15.

Wiley, K., Lefebvre, K. B., Wall, L., Baldwin-Medsker, A., Nguyen, K., Marsh, L., \& Baniewicz, D. (2017). Immunotherapy administration: oncology nursing society recommendations. Clinical Journal of Oncology Nursing, 21(2), 2-7. 
Research, Society and Development, v. 10, n. 7, e46910716871, 2021

(CC BY 4.0) | ISSN 2525-3409 | DOI: http://dx.doi.org/10.33448/rsd-v10i7.16871

World Health Organization. (2020). WHO report on cancer: setting priorities, investing wisely and providing care for all care for all. WHO.

Ysebaert, L., Larcher, M., Compaci, G., Oberic, L., Sahnes, L., Banos, A., Araujo, C., Sommet, A., Laurent, G., \& Despas, F. (2019). Oncology nurse phone calls halve the risk of reduced dose intensity of immunochemotherapy: results of the randomized FORTIS study in chronic lymphocytic leukemia. Annals of Hematology, 98(4), 931-939. 